\title{
PsSAK1, a Stress-Activated MAP Kinase of Phytophthora sojae, Is Required for Zoospore Viability and Infection of Soybean
}

\author{
Aining Li, Yonglin Wang, Kai Tao, Suomeng Dong, Qian Huang, Tingting Dai, Xiaobo Zheng, and \\ Yuanchao Wang
}

Department of Plant Pathology, Nanjing Agricultural University, Nanjing 210095, China

Submitted 1 January 2010. Accepted 15 April 2010.

\begin{abstract}
Mitogen-activated protein kinase (MAPK) pathways are universal and evolutionarily conserved signal transduction modules in all eukaryotic cells. In this study, PsSAK1, which encodes a stress-activated MAPK of Phytophthora sojae, was identified. PsSAK1 is highly conserved in oomycetes, and it represents a novel group of MAPK due to its pleckstrin homology domain. Reversetranscription polymerase chain reaction analysis showed that PSSAK1 expression was upregulated in zoospores and cysts and during early infection. In addition, its expression was induced by osmotic and oxidative stress mediated by $\mathrm{NaCl}$ and $\mathrm{H}_{2} \mathrm{O}_{2}$, respectively. To elucidate the function, the expression of PSSAK1 was silenced using stable transformation of $P$. sojae. The silencing of PSSAKI did not impair hyphal growth, sporulation, or oospore production but severely hindered zoospore development, in that the silenced strains showed quicker encystment and a lower germination ratio than the wild type. PsSAK1-silenced mutants produced much longer germ tubes and could not colonize either wounded or unwounded soybean leaves. Our results indicate that PsSAK1 is an important regulator of zoospore development and pathogenicity in $P$. sojae.
\end{abstract}

Oomycetes, fungus-like microorganisms that are evolutionarily related to marine algae (Förster et al. 1990; Sogin and Silberman 1998; Harper et al. 2005; Tyler et al. 2006), include a variety of plant and animal pathogens responsible for many economically important diseases. The genus Phytophthora includes devastating pathogens of many cultivated plants (Erwin and Ribiero 1996). Notorious members of this genus include Phytophthora infestans, the potato late blight pathogen responsible for the Irish potato famine; $P$. ramorum, the cause of sudden oak death; and the soybean root and stem rot pathogen $P$. sojae. Root rot caused by $P$. sojae results in $\$ 200$ to 300 million in annual losses in the United States, and approximately $\$ 1$ to 2 billion in annual losses worldwide (Wrather and Koenning 2006). P. sojae is a threat to soybean production all over the world, and current control methods are inadequate.

Aining Li and Yonglin Wang contributed equally to this work.

Corresponding author: Y. Wang; Telephone: +86-25-84399071; Fax: +8625-84395325; E-mail: wangyc@ njau.edu.cn

* The $\boldsymbol{e}$-Xtra logo stands for "electronic extra" and indicates that five supplementary figures and seven supplementary video presentations are available online. Also, Figure 7 appears in color online.
$P$. sojae produces heterokont, biflagellate zoospores that are released from sporangia into water. The zoospores develop by cytokinesis of multinucleate sporangia in asexually sporulating cultures and are able to swim toward the roots of host plants (Tyler 2007). Free-swimming zoospores lack cell walls and, thus, their plasma membrane is directly exposed to the environment. Once reaching potential host plants, the zoospores rapidly transform into adhesive cysts with cellulosic cell walls. Infection occurs when cysts germinate to produce hyphae or form appressoria. The hyphae may directly penetrate the plant epidermis, sometimes forming appressoria (Enkerli et al. 1997; Tyler 2007). P. sojae zoospores are chemotactic toward nutrients and other molecules released by soybean roots such as isoflavones (Tyler et al. 1996; Morris et al. 1998). Previously, the motility and virulence of zoospores was demonstrated to be mediated by a G- $\alpha$-protein in P. sojae (Hua et al. 2008) and $P$. infestans (Latijnhouwers et al. 2004). However, the molecular mechanisms that control zoospore development and behavior are still largely unknown in $P$. sojae. In $P$. infestans, a calciumand calmodulin-regulated protein kinase is known to be induced during zoosporogenesis (Judelson and Roberts 2002), and a bZIP transcription factor that interacts with a protein kinase is required for zoospore motility and plant infection (Blanco and Judelson 2005). A DEAD-box RNA-helicase gene that is highly expressed in zoospores is required for normal zoospore development (Walker et al. 2008). The involvement of phospholipase D (PLD) in controlling zoospore behavior has also been proposed because phosphatidic acid, a messenger molecule generated by hydrolysis of structural phospholipids by PLD, induces zoospore encystment (Latijnhouwers et al. 2002).

Accumulating evidence implicates mitogen-activated protein kinase (MAPK) signaling pathways in the control of zoospore development and behavior. In fungi, the MAPK pathways are known to function downstream of the heterotrimeric G-protein complex (Li et al. 2007). MAPK are important signal transduction molecules involved in many facets of cellular regulation, such as cell division, differentiation, cell survival, and apoptosis in response to diverse extracellular stimuli (Herskowitz 1995; Treisman 1996; Banuett 1998; Gustin et al. 1998). MAPK that specifically transmit environmental stress signals are known as stress-activated protein kinases (SAPK). Members of SAPK include Saccharomyces cerevisiae Hog1, which is integral to the osmoregulatory signal transduction cascade (HOG signaling pathway; loss of Hog $1 p$ activity results in reduced growth on high-osmolarity media, abnormal cell and budding morphology, and osmolarity-induced activation of the pheromone response pathway) 
(Brewster et al. 1993; Gustin et al. 1998); S. spombe Sty1 (also called Spc1), which is activated following exposure to high-osmolarity media or growth in suboptimal nutrient conditions (Degols et al. 1996); Magnaporthe grisea Osm1, in which mutants are sensitive to osmotic stress and show morphological defects when grown under hyperosmotic conditions (Dixon et al. 1999); and mammalian p38s and JNKs, which are involved in the inflammatory and stress responses (Kyriakis and Avruch 2001). To adapt to new conditions, different strategies involving diverse regulatory mechanisms have evolved in fungi. In $S$. cerevisiae, Hog 1 is activated by hyperosmotic stress (Brewster et al. 1993; Schuller et al. 1994; Winkler et al. 2002). Sty1 and mammalian SAPK are responsible for the response to a wide range of stresses, such as hyperosmolarity, heat shock, UV light irradiation, and oxidative stress (Millar et al. 1995; Shiozaki and Russell 1995; Waskiewicz and Cooper 1995; Degols et al. 1996; Kato et al. 1996; Kyriakis and Avruch 1996; Hohmann 2002). The deletion of SAPK in fungi results in impairment of osmoregulation and the stress response. For example, in $S$. cerevisiae, hogl mutants are only sensitive to high osmolarity, whereas styl mutants are also sensitive to heat shock and oxidative stress. Moreover, Osm 1 of M. grisea controls the accumulation of arabitol in response to hyperosmolarity in hyphae but not the accumulation of glycerol for generation of turgor in appressoria. In addition, the deletion of $O s m 1$ does not affect virulence (Dixon et al. 1999). In Cryphonectria parasitica, knockout of cpmkl impairs pigmentation, conidiation, laccase production, and virulence (Park et al. 2004). In Mycosphaerella graminicola, deletion of $M g h o g l$ leads to osmosensitivity, preventing transition from yeast-like growth to filamentous growth and impairing pathogenicity (Mehrabi et al. 2006). In Bipolaris oryzae, the Hog1-type MAPK homolog SRM1 gene deletion shows growth inhibition under hyperosmotic, hydrogen peroxide, and UV exposure conditions (Moriwaki et al. 2006). Therefore, the role of SAPK in filamentous fungi is markedly divergent.

In a transcript profiling analysis using the mRNA sequencing method, which is a recently developed approach to transcriptome profiling that uses deep-sequencing technologies, we identified a MAPK gene and named it PSSAK1, which is significantly upregulated in zoospores and cysts. Bioinformatics studies indicate that this gene encodes a MAPK with a high homology to a stress-activated MAPK, S. cerevisiae Hog1. PsSAK1-silenced lines display altered zoospore development, including premature encystment and reduced germination. Heightened sensitivity to $\mathrm{NaCl}$ treatment and reduced infection toward soybean were other features of the PSSAKI-silenced lines compared with the wild-type (WT) strain and the control (CK) transformant.

\section{RESULTS}

\section{PSSAK1 gene encodes a stress-activated MAPK with a pleckstrin homology domain.}

From a global transcript profiling analysis (Solexa mRNA sequence) (data not yet published), a gene highly transcribed in the stages of zoospore and cyst was identified; therefore, we performed an advanced search in the $P$. sojae genome and found that it encoded a predicted MAPK (protein ID: 144802). The amino acid sequence of the predicted open reading frame (ORF) showed high similarity to Hog1 (38\% identity) from $S$. cerevisiae and was also similar to other SAPK such as Osm1 (43\% identity) in Magnaporthe grisea, Sty1 (45\% identity) in Schizosaccharomyces pombe, and $\mathrm{p} 38 \alpha$ (43\% identity) in Homo sapiens (data not shown). The predicted ORF is 2,106 bp in length, without an intron, and encodes a protein with 702 amino acids. The predicted ORF had three expressed sequence tag (EST) records (psHB043xC11f, psMA002xP05f, and psZS014xN12f) in the VBI Phytophthora EST database, indicating that the gene model was correctly predicted; the model was also supported by reverse-transcription polymerase chain reaction (RT-PCR) results (data not shown). Based on the sequence similarity, we named this ORF the $P$. sojae stress-activated protein kinase $(P S S A K 1)$ (GenBank accession number FJ349603).

Characteristic conserved subdomains of MAPK could be identified in the PsSAK1 sequence, including the dual phosphorylation lip "TEY" (at positions 399 to 401) and the MAPK signature sequence [LIVM][TS]XX[LIVM]XT[RK] [WY]YR XPX[LIVM][LIVM] (Kultz 1998). The alignment of PsSAK1 with other MAPK revealed similarity in the MAPK domain, including the Thr-X-Tyr motif in the activation loop, ATPbinding pocket, and catalytic loop (data not shown). However, a notable difference between PsSAK1 and other known MAPK is the presence of a pleckstrin homology $(\mathrm{PH})$ domain in its $\mathrm{N}$ terminus (Supplementary Fig. S1). This would be appear to be a novel feature, because no other MAPK has been reported that contains a PH domain.

To test whether unexpected copies or paralogs exist in the whole-genome sequence, we used EcoRI-, EcoRV-, or HindIIIdigested $P$. sojae (P6497) genomic DNA (gDNA) to hybridize with the specific probe of PsSAK1. Southern blotting showed that PsSAKl was a single-copy gene in P. sojae and did not have any highly similar paralogs (Supplementary Fig. S2). PsSAK1 is highly conserved among sequenced oomycete genomes, including $P$. ramorum, $P$. infestans, $P$. capsici, and Hyaloperonospora arabidopsidis. These orthologs were named PrSAK1, PiSAK1, PcSAK1, and HpSAK1, respectively. Phylogenetic comparisons with published MAPK genes revealed that the oomycete SAK1s group as a unique clade that belongs to the stress-activated MAPK subfamily, including MAPK from humans, plants, and fungi (Supplementary Fig. S3). Taken together, these results indicate that PsSAK1 and other oomycete SAK1s would be members of the stress-activated MAPK subfamily.

\section{PSSAK1 is highly expressed}

in zoospores and infection sites and is stress responsive.

To determine the expression patterns of PsSAk1 during distinct developmental life stages, including vegetative hyphae, sporulating hyphae, zoospores, cysts, and germinated cysts, and during infection, we employed RT-PCR to analyze mRNA accumulation. Measurement of PSSAK1 expression in various life-cycle stages of $P$. sojae indicated that this transcript could be detected in all cell types tested, although it was most abundant in zoospores and cysts (Fig. 1A). Meanwhile, expression of PsSAK1 was also detected during plant infection, with maximum expression occurring at $0.5 \mathrm{~h}$ postinoculation (hpi). During the later infection stages, at 8 and $12 \mathrm{hpi}$, the mRNA of PSSAK1 is barely detectable (Fig. 1B). These results were identical to the expression profiling defined by the RNA-Seq technology (unpublished data).

To determine whether PSSAK1 is a stress-activated gene, we investigated its expression pattern under oxidative and osmotic stress. Treatment with $\mathrm{H}_{2} \mathrm{O}_{2}$ has been used to mimic the oxidative stress that Phytophthora spp. encounter while infecting hosts (Yoshioka et al. 2003; Chen et al. 2008), and $\mathrm{NaCl}$ is used as an osmotic or salt stress in fungi (Shen et al. 2001; Hohmann 2002). Under stress mediated by $\mathrm{H}_{2} \mathrm{O}_{2}$ or $\mathrm{NaCl}$ treatment, the expression of $P S S A K 1$ noticeably increases (Fig. 1C). We also found that the expression level of PSSAK1 is positively correlated with the concentration and the treatment time of $\mathrm{H}_{2} \mathrm{O}_{2}$ (Fig. 1C). 
Transformation with antisense constructs results in silencing of PSSAK1 expression.

To explore the function of PSSAK1 and to determine whether loss of this gene affects $P$. sojae development, response to stress, or pathogenicity, we silenced the gene using a method based on polyethylene glycol (PEG)-mediated protoplast stable transformation (Hua et al. 2008). We co-transformed $P$. sojae protoplast cells with a construct containing the PSSAKI coding region in an antisense orientation driven by the constitutive pHam 34 promoter, together with the pTH209 vector carrying the geneticin resistance gene for selection (Judelson et al. 1991) (Fig. 2A). In total, 143 putative transformants that could grow in the presence of geneticin at $50 \mu \mathrm{g} / \mu \mathrm{l}$ were selected for further assay. Of 143 putative PsSAK1 transformants, 67 PsSAK1-integrated transformants were obtained using PCR (data not shown). Subsequently, these were used to evaluate the level of PSSAKI mRNA accumulation by RTPCR. Of 67 PsSAK1-integrated transformants, only two transformants (T31 and T61) failed to produce the given amplicon when the normal number of PCR cycles was applied with germinated cyst RNA as initial template (Fig. 2B). Finally, Southern blot analysis of the two PsSAKI-silenced lines revealed that extra multi-copies of PSSAKI had been integrated into the genome of the transformants (Fig. 2C). Thus, we successfully obtained two independent $P$. sojae lines deficient in $P S S A K 1$ expression through stable transformation with an antisense gene construct.

\section{PsSAK1-silenced transformants show aberrant} zoospore development and less osmotic adaptation.

Compared with WT recipient strain P6497 and a CK strain expressing only the $\beta$-glucuronidase reporter gene, the PsSAKI-
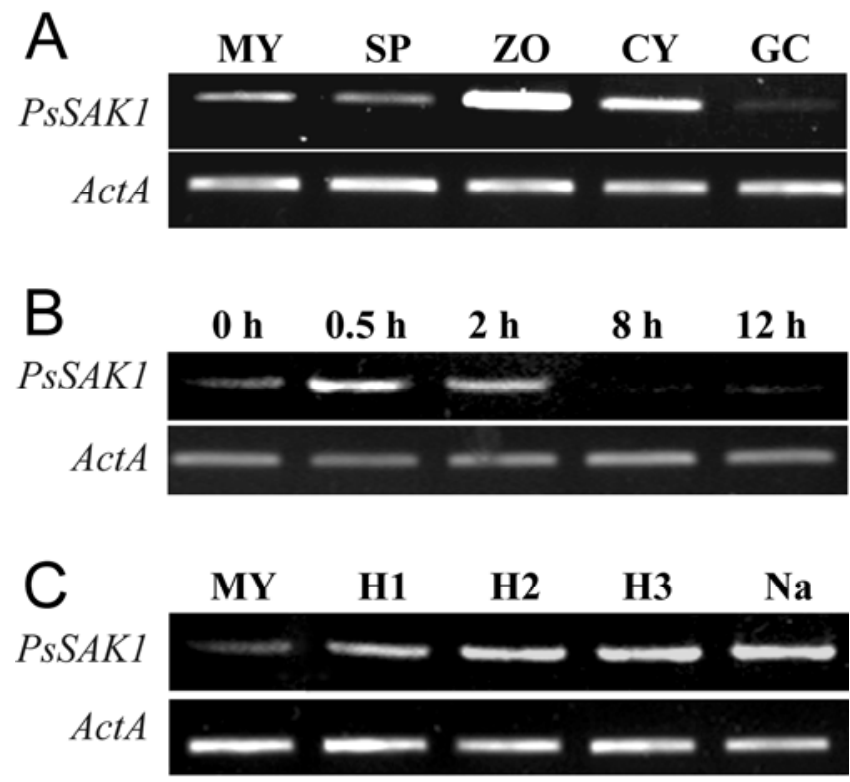

Fig. 1. Expression analysis of PsSAK1. A, Expression of PsSAK1 during asexual development by using reverse-transcription polymerase chain reaction (RT-PCR) detection. RNA samples are from Phytophthora sojae strains P6497 vegetative hyphae (MY), sporulating hyphae (SP), zoospores (ZO), cysts (CY), and germinating cysts (GC). B, Expression of PSSAK1 during early stage of the compatible interaction. P6497 mycelium are inoculated on soybean cv. Williams leaves at $0.5,2,8$, and 12 h postinoculation. Total RNA was then extracted for RT-PCR analysis. C, Expression of PsSAK1 under $\mathrm{H}_{2} \mathrm{O}_{2}$ and $\mathrm{NaCl}$ stresses. Mycelia are cultured in liquid $10 \%$ V8. RNA samples from mycelium washed with sterile distilled water three times (MY), treated with $1 \mathrm{mM} \mathrm{H}_{2} \mathrm{O}_{2}$ for 10 $\min (\mathrm{H} 1)$ or $30 \mathrm{~min}(\mathrm{H} 2)$, or treated with $4 \mathrm{mM} \mathrm{H}_{2} \mathrm{O}_{2}$ for $10 \mathrm{~min}(\mathrm{H} 3)$ and $0.2 \mathrm{M} \mathrm{NaCl}$ for $30 \mathrm{~min}(\mathrm{Na})$ was analyzed by using RT-PCR. silenced lines T31 and T61 displayed no differences in growth rate or in oospore or zoospore production (Table 1). Also, no differences in cell or hyphal shape could be observed (data not shown).

However, zoospore behavior was severely affected by silencing PsSAK1. First, when zoospores were released from sporangia of PSSAK1-silenced lines, they appeared as less-active, obese protoplasmic balls, and were not as bean-shaped as zoospores of P6497. Nearly $8 \%$ zoospores of PSSAK1-silenced lines were abnormal and, in each of them, two incompletely separated zoospores seemed to be stuck together. The second striking difference was that approximately $20 \%$ of zoospores of $P S S A K 1$-silenced lines burst immediately after they were released from sporangia but almost none of the zoospores from P6497 and the CK ruptured. Note that, whereas sporangia of PsSAK1-silenced lines released zoospores into $0.05 \mathrm{M}$ mannitol solution, it was hard to find ruptured zoospores. Little effect on zoospores of P6497 and CK was observed at this mannitol concentration (Fig. 3) (Supplementary Video S1). When increasing the concentration, these zoospores became bean-shaped again and their mobility immediately recovered to a certain ex-

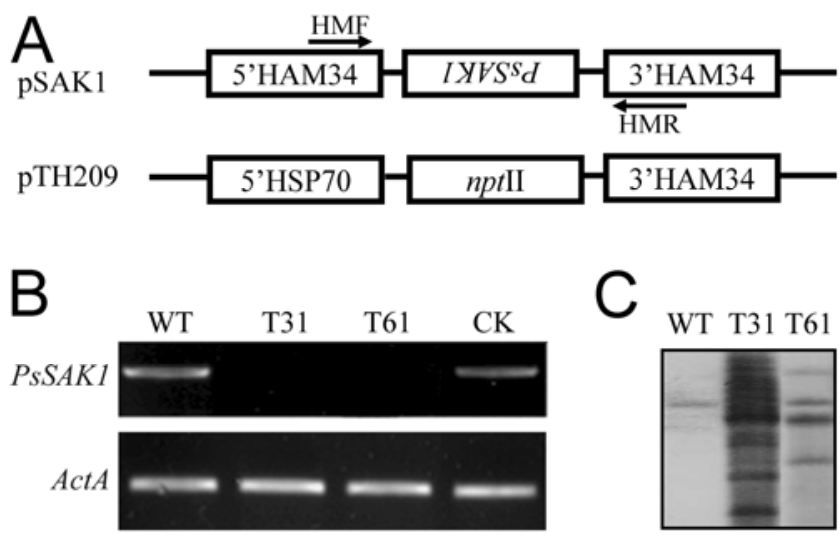

Fig. 2. Generation of PsSAKl-silenced lines. A, Constructs used for Phytophthora sojae transformation. Expression cassettes are shown. Plasmid pSAK1 consists of the full length of PsSAK1 in antisense orientation and $n p t$ II is the neomycin phosphotransferase II gene used as screen marker for co-transformation. 5'HAM34 and 5'HSP70 represent promoters, with $3^{\prime}$ HAM34 as terminator. HMF and HMR indicate the primers used for positive-transformation polymerase chain reaction (PCR) screening. B, Reverse-transcription (RT)-PCR evaluation of PsSAK1 silencing efficiency. Expression of PSSAK1 in mycelium of the wild-type strain P6497 (WT), PsSAK1-silenced mutants (T31 and T61), and the transformant expressing $\beta$-glucuronidase $(\mathrm{CK})$. C, Southern blot analysis. Genomic DNA from WT, T31, and T61 was digested with EcoRI. Partial coding region of $P S S A K 1$ was used as a probe.

Table 1. Phenotypic characteristics of PsSAK1-silenced transformants

\begin{tabular}{lccc}
\hline $\begin{array}{l}\text { Strain or } \\
\text { transformant }^{\mathbf{a}}\end{array}$ & $\begin{array}{c}\text { Growth rate } \\
(\mathbf{m m} / \mathbf{d a y})^{\mathbf{b}}\end{array}$ & ${\text { Oospore } / \boldsymbol{\mu \mathbf { l } ^ { \mathbf { c } }}}$ & Zoospore $/ \boldsymbol{\mu l}^{\mathbf{d}}$ \\
\hline WT & $4.58 \pm 0.08$ & $5.47 \pm 0.63$ & $41.10 \pm 3.42$ \\
CK & $4.42 \pm 0.13$ & $5.83 \pm 0.81$ & $39.11 \pm 1.89$ \\
T31 & $4.48 \pm 0.15$ & $5.82 \pm 0.67$ & $38.93 \pm 4.35$ \\
T61 & $4.42 \pm 0.08$ & $5.22 \pm 0.91$ & $44.15 \pm 2.50$ \\
\hline
\end{tabular}

${ }^{a}$ All transformants were generated using P6497 as the recipient strain. For each characteristic, experiments were independently repeated more than two times, and three cultures from each lines were tested in each experiment. $\mathrm{WT}=$ wild type and $\mathrm{CK}=$ control.

${ }^{\mathrm{b}}$ Growth rate was calculated on $10 \%$ V8 juice agar medium for 4 days.

${ }^{c}$ Number of oospores was counted in $10 \%$ V8 juice liquid medium for 20 days.

${ }^{\mathrm{d}}$ Based on 3 days of growth in 10\% V8 juice liquid medium followed by inducing zoosporogenesis, the number of zoospores was counted under the microscope. 
tent and then crashed soon after due to the high extracellular osmolarity. The third obvious difference was that the time interval between zoospore release and encystment of PSSAK1silenced lines was much shorter than that of the WT and CK transformants. Most of these zoospores just subsided instead of swimming away as quickly as possible like normal zoospores of P6497. The results indicate that 84 and $76 \%$ of zoospores from the PSSAK1-silenced lines T31 and T61, respectively, were encysted within $0.5 \mathrm{~h}$ after release from sporangia, whereas such a high level of encystment was not reached until at least 6 $\mathrm{h}$ in the WT and CK transformant (Fig. 4A). Virtually all zoospores were encysted after $8 \mathrm{~h}$, regardless of the $P$. sojae strain tested.

Under optimal conditions, once zoospores encyst, they start to germinate and produce hyphae or appressoria in response to physical and chemical signals (Morris et al. 1998; Zuo et al. 2005). Here, we tested whether the silencing of PSSAKI affected cyst germination. Germination was measured by vigorously shaking a zoospore suspension, followed by transferring drops of the resulting cyst suspension onto glass plates. The encysted zoospores were incubated in a growth medium and the germination ratio was recorded over a 12-h time course. The results indicate that cyst germination is severely reduced by silencing of $P S S A K 1$ based on a two-tailed $t$ test $(P<0.01)$ (Fig. 4B).

To further test the ability of germinated cysts to form appressoria, zoospore suspensions were placed on soybean leaves. After $4 \mathrm{~h}$, approximately $33 \%$ of the germinated cysts of the WT were observed to produce appressoria (Fig. 5A). In contrast, $<5 \%$ of the germinating cysts from the PsSAK1silenced line T31 produced normal appressoria. Furthermore, germ tubes of T31 germinating cysts were longer than those of the WT, and the tips only showed slight swelling (Fig. $5 \mathrm{~B})$.

\section{PsSAK1-silenced transformants are sensitive to NaCl.}

RT-PCR analysis of PsSAK1 has demonstrated that it is associated with the response to stresses such as salt and oxidative stress. In addition, in several lines, disruption of SAPK has been reported to affect the response to a wide range of stresses (Shiozaki and Russell 1995; Waskiewicz and Cooper 1995; Degols et al. 1996; Kyriakis and Avruch 1996; Hohmann 2002). To determine any differences in the PSSAKI-silenced transformants, the response to osmotic stress mediated using $\mathrm{H}_{2} \mathrm{O}_{2}$ and $\mathrm{NaCl}$ was examined. Although the PsSAKI-silenced transformants did not show any alteration in growth characteristics under stress mediated with $\mathrm{H}_{2} \mathrm{O}_{2}$ (data not shown), growth in the presence of $0.2 \mathrm{M} \mathrm{NaCl}$ was suppressed compared with the WT and CK strains. Both WT strains and mutants were measured and compared on solid media with $0.2 \mathrm{M} \mathrm{NaCl}$ with no significant difference in linear growth rate. However, the density of mycelium in the mutant colony is significantly lower than in the CK colony, suggesting that the biomass of the colony is different. Therefore, to measure and describe this phenotype, we conducted the liquid media culture and measured the dry weight. After 5, 7, 9, and 11 days of incubation in liquid Plich medium (without additions) or with $0.2 \mathrm{M}$ added $\mathrm{NaCl}$, the dry mass of PSSAK1 nonsilenced strains (WT and $\mathrm{CK}$ ) and PsSAK1-silenced strains (T31 and T61) was determined. The results show that the PsSAK1-silenced strains were growth-impaired in the presence of $0.2 \mathrm{M} \mathrm{NaCl}$ compared with the WT and CK strains (Fig. 6), indicating that growth of the silenced transformants is sensitive to $\mathrm{NaCl}$. Incubation in liquid Plich medium with an additional $0.4 \mathrm{M} \mathrm{NaCl}$ resulted in no growth of any of the four tested $P$. sojae strains (T31, T61, WT, and CK).

\section{PsSAK1 is essential for plant infection.}

Silencing of PSSAK1 also affected pathogen pathogenicity. Virulence was tested by pipetting zoospore suspension onto soybean leaves, with or without prewounding the inoculation sites. Comparison of the two nonsilenced strains, WT and CK, to the two silenced lines, T31 and T61, revealed striking differences. At 3 days postinoculation (dpi) with zoospores of the

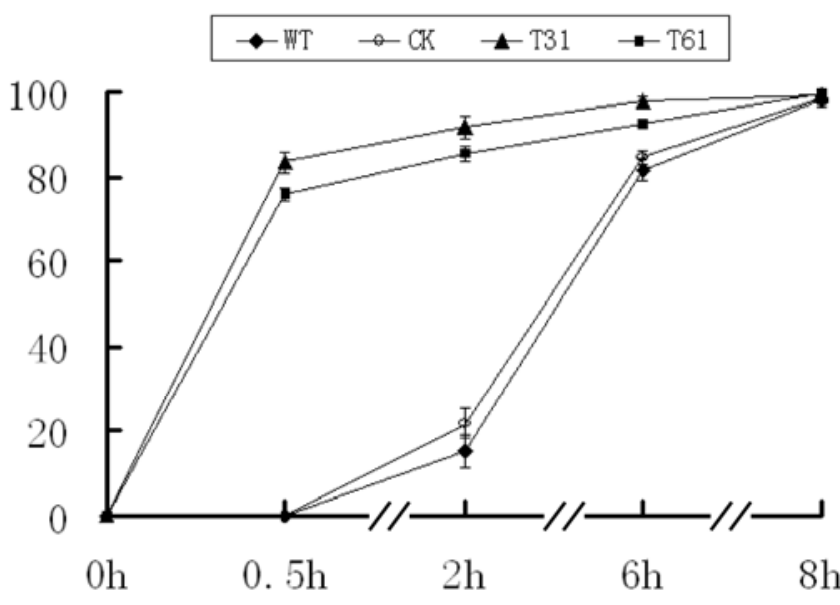

Fig. 4. Zoospore abnormal development in PsSAK1 silencing line. A, Zoospore encystment rates was examined at $0,0.5,2,6$, and $8 \mathrm{~h}$ postzoospore release. Experiments were repeated twice. B, Cyst germination rates. Numbers of germinated cysts and nongerminated cysts were counted and calculated at 0, 2, 4, 6, and $12 \mathrm{~h}$ after encyst treatment. Experiments were conducted three times.
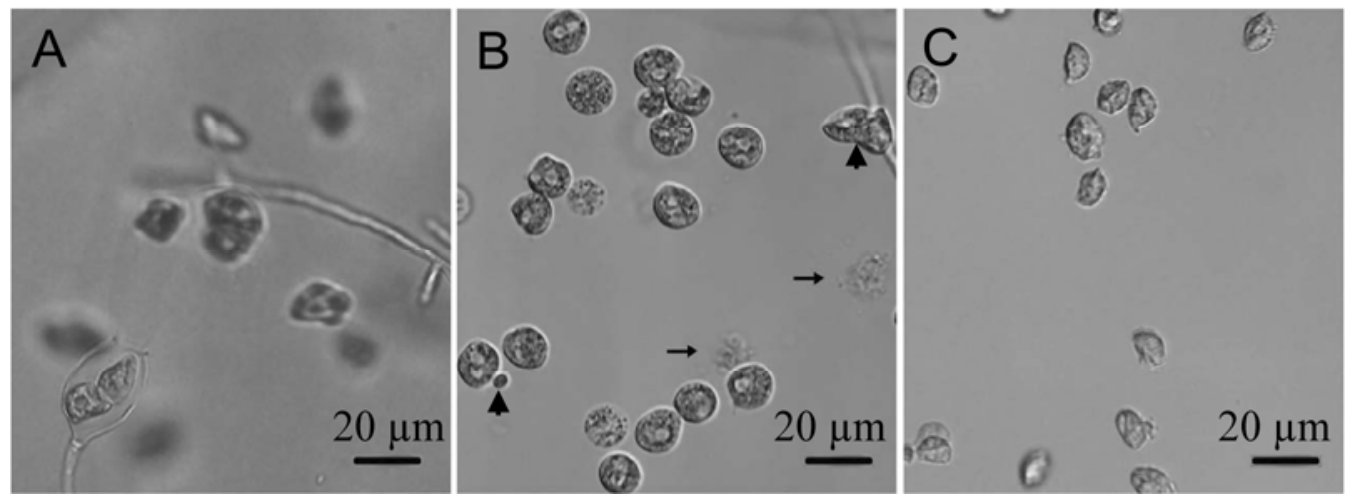

Fig. 3. Microscopic observations of swimming zoospore. A, Normal bean-shaped zoospores from the wild type. B, Zoospores from PsSAK1-silenced line T31. Abnormal and burst zoospores were shown in arrowhead and arrow, respectively. C, Morphology and mobility of zoospores from PsSAK1-silenced line T31 was recovered at a high concentration of sorbitol. 
WT and CK strains, the leaves displayed water-soaked lesions, a typical disease symptom caused by $P$. sojae. At 5 dpi, the lesions had spread all over the leaves (Fig. 7A). The disease symptoms were more severe on wounded leaves than on nonwounded leaves. The $P$. sojae WT and $\mathrm{CK}$ infections on wounded leaves produced masses of aerial hyphae by 5 dpi. In contrast, neither of the two silenced lines, T31 and T61, pro-
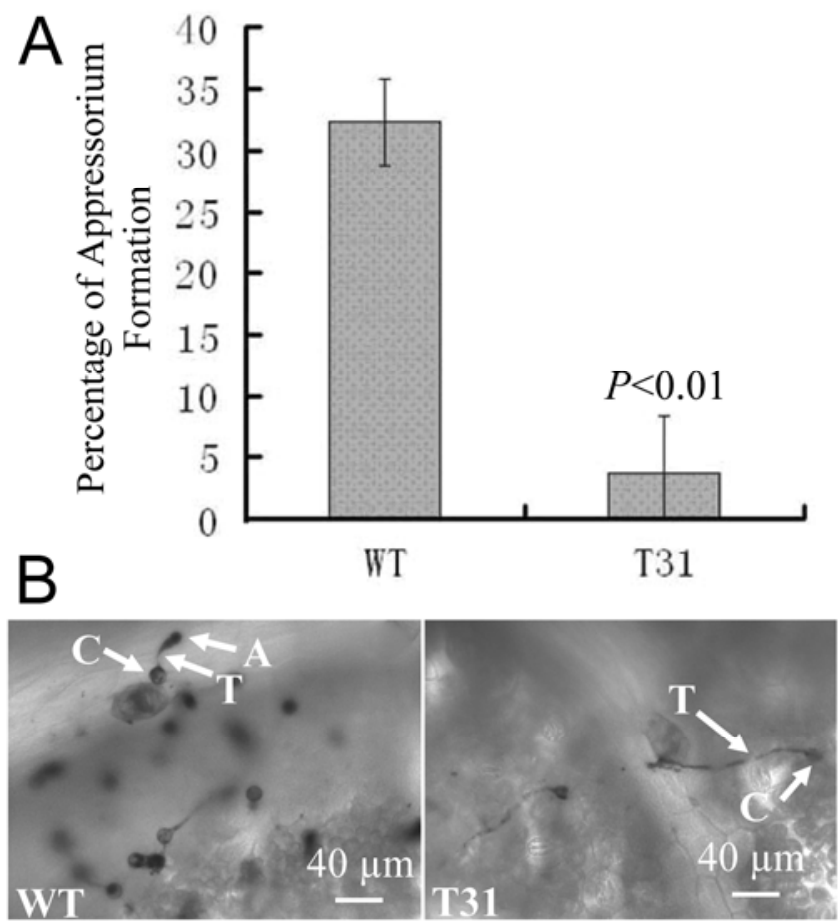

Fig. 5. Appressorium formation of PsSAK1-silenced transformants was reduced. A, Appressorium formation ratios on soybean leaves were counted and compared. At least 100 cysts were observed for the wild type (WT) and T31, respectively. B, Assay for appressorium formation on soybean leaves was observed under microscope. The cyst (C), germ tube (T), and appressorium (A) are labeled for one representative propagule. Bars at the base of the panels equal $40 \mu \mathrm{m}$.

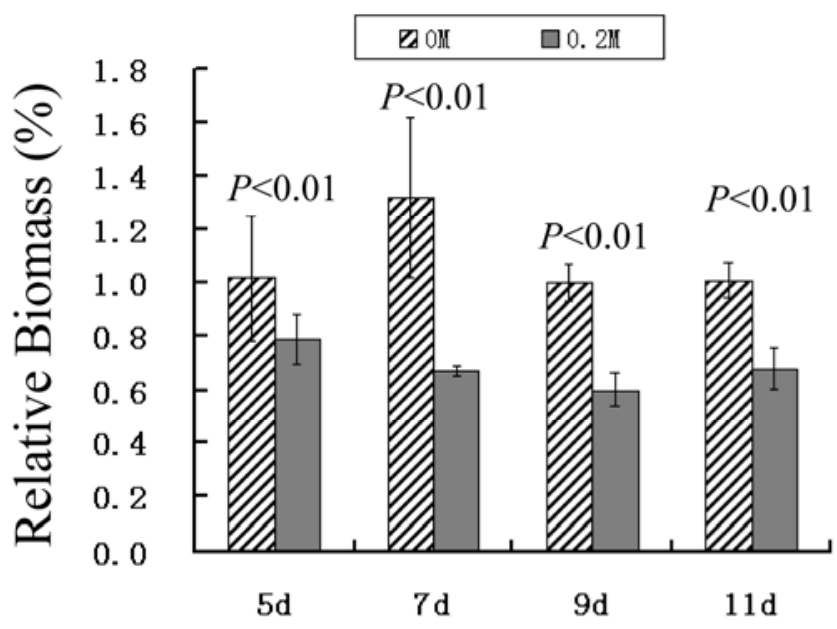

Fig. 6. Effect of silencing PSSAK1 on the tolerance against osmotic stress. Relative biomass based on the dry weight of mycelia from wild-type (WT), control (CK), T31, and T61 strains cultured in Plich's liquid medium supplemented with $0 \mathrm{M}$ and $0.2 \mathrm{M} \mathrm{NaCl}$ for 5, 7, 9, and 11 days, respectively. Mean of the dry weight from T31 and T61 divided by the mean of the dry weight from WT and CK represented the relative biomass. Experiments were repeated twice and three cultures were tested in each experiment. duced any visible disease symptoms at 3 dpi. At 5 dpi, plant cell necrosis was visible, especially in the wounded leaves, but this was limited to the site of wounding and inoculation. No evidence of successful $P$. sojae infection, such as spreading,
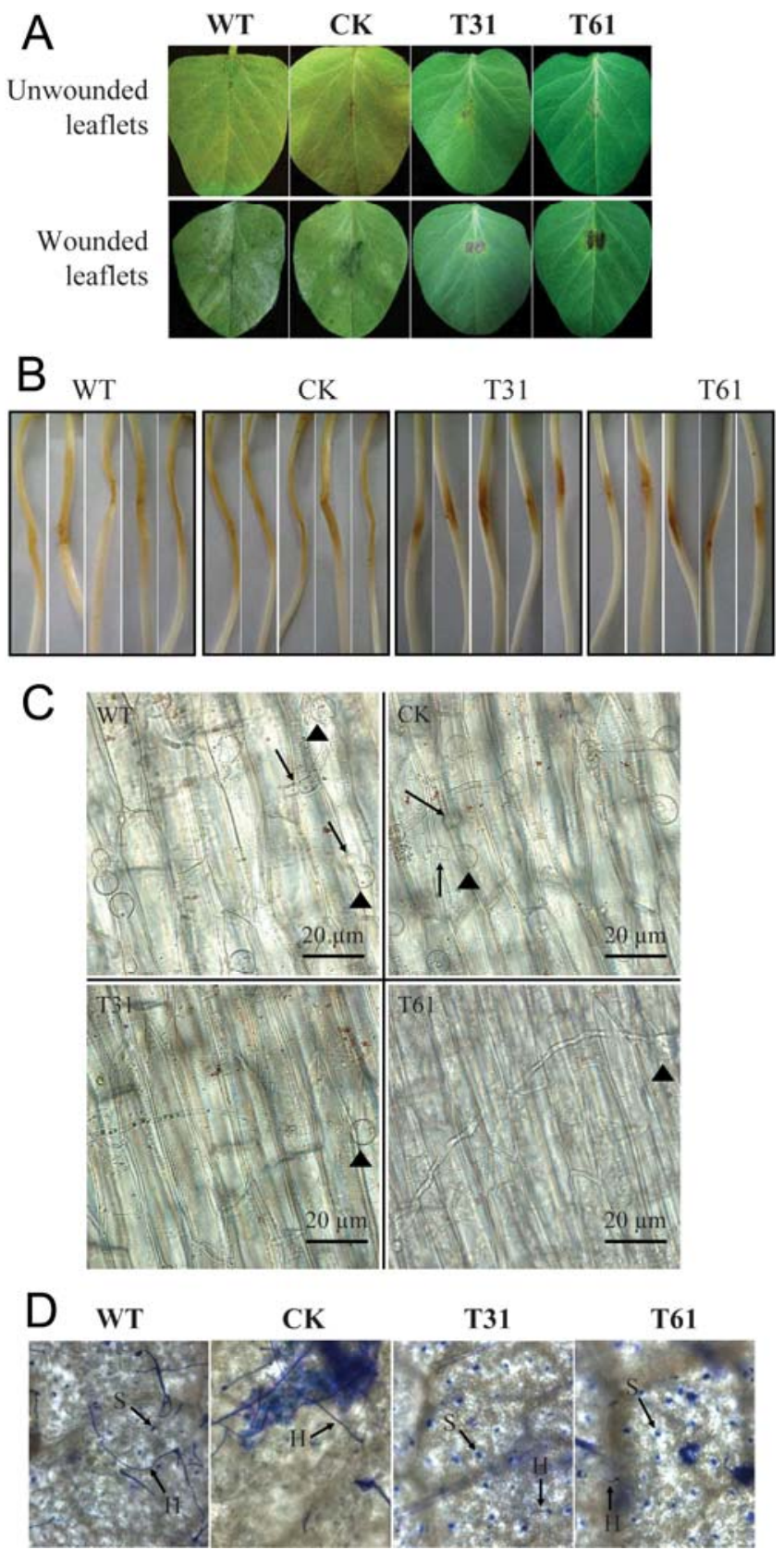

Fig. 7. PSSAK1-silenced mutants showed reduced virulence. A, Unwounded and wounded leaflets from 15-day-old Williams soybean (susceptible) were spot incubated with equal numbers of zoospores. Photographs were taken at 5 days postinoculation. WT, wild-type strain P6497; CK, the transformant expressing the $\beta$-glucuronidase; T31 and T61 are PsSAK1-silenced lines. B, Etiolate soybean seedlings were inoculated with hyphal tip plugs from P6497-, CK-, and PsSAK1-silenced mutants (T31 and T61) for $36 \mathrm{~h}$. Experiments were repeated at least four times in independent occasions. C, Microscopic observation of the infection of germinated cysts on the surface of hypocotyls. Etiolate soybean seedling were inoculated with zoospore suspensions from P6497-, CK-, and PSSAK1-silenced mutants (T31 and T61) for $6 \mathrm{~h}$. Then, the epidermis tissues around inoculation site were peeled off and used for microscopic observation. Cysts and infection sites were shown in arrowhead and arrow, respectively. D, Microscopic observation of infection hyphae on leaves. Leaves were infected by the equal amount of zoospores from WT, CK, T31, and T61 for $24 \mathrm{~h}$. H, hypha and S, stoma. 
water-soaked lesions, or growth of aerial hyphae, was observed in the PsSAK1-silenced strains T31 and T61 (Fig. 7A). The same results were obtained when unwounded and wounded soybean hypocotyls were inoculated with zoospore suspensions from PSSAK1-silenced and nonsilenced strains (Supplementary Fig. S4).

To further confirm the loss of virulence of T31 and T61, etiolated soybean hypocotyls inoculated with hyphal plugs, as described by Qutob and associates (2002), were also used in this research. This hypocotyl inoculation test produced results similar to the leaf- and zoospore-based assay (Fig. 7B). At 36 hpi, hypocotyls inoculated with hyphal plugs of P6497 or CK displayed long, water-soaked lesions, whereas hypocotyls inoculated with hyphae of T31 and T61 showed short, darkbrown necrosis. These results indicate that silencing of PsSAK1 led to pathogenicity loss of $P$. sojae for zoospore- or hyphae-based inoculation of leaves or hypocotyls, respectively.

To explore the reason for the pathogenicity defect in PsSAK1-silenced lines, a microscopic examination of infection was performed. The germinated cysts of the PSSAK1-silenced mutants produced much longer germ tubes on the surface of the epidermis than the WT or CK strain, and the germ tubes of mutants could not penetrate host hypocotyl epidermal cells (Fig. 7C). The microscopic observation of zoospore-inoculated leaves also suggested that germ tubes of cysts from the two silenced lines failed to penetrate host leaf epidermal cells. Therefore, most of the hyphae were washed away after washing leaves repeatedly. In contrast, the hyphae from germinating cysts of WT and CK were able to invade tissues of leaves and were not easily washed off (Fig. 7D). The infection ability was also tested on onion epidermal cells. The WT and a CK transformant could infect epidermal cells and expand into the epidermal tissues, whereas PSSAK1-silenced mutants (T31 and T61) could not infect epidermal cells even though the mutants produced several appressoria (Supplementary Fig. S5). These results indicate that the silencing of PSSAK1 not only impaired zoospore germination but also severely affected the ability of germ tubes or hyphae of the germinated cysts to penetrate the host tissues.

\section{DISCUSSION}

In this article, we report that a stress-activated MAPK from P. sojae, PSSAK1, controls zoospore development and is necessary for pathogenicity. PsSAK1 contains a pleckstrin homology domain in the N-terminal region, and the primary structure of this protein is conserved in all oomycete genomes sequenced to date. $P S S A K 1$ is differentially expressed during the $P$. sojae life cycle and is upregulated by stress and during early infection.

\section{PsSAK1 represents a novel MAPK.}

MAPK represent a special type of protein kinase that is highly conserved and present only in eukaryotic cells (Kultz 1998; Roman et al. 2007). We identified PsSAK1 from the $P$. sojae genome using HOG1 of yeast and other fungal SAPK as query sequences in BLAST searches. The orthologs of PsSAK1 were identified in other sequenced Phytophthora spp. and in the $H$. arabidopsis genome. The oomycete SAK1s all contain $\mathrm{PH}$ domains at the $\mathrm{N}$-terminal region of the protein. The $\mathrm{PH}$ domain occurs in a wide range of proteins involved in intracellular signaling or as constituents of the cytoskeleton but we know of no previous report describing a MAPK with a PH domain (Ingley and Hemmings 1994; Rebecchi and Scarlata 1998). PH domains are found in cellular signaling proteins such as serine/threonine kinase, tyrosine kinases, regulators of G-proteins, endocytotic GTPases, and adaptors, as well as cytoskeletal-associated molecules and lipid-associ- ated enzymes. This domain family possesses multiple functions, including the subcellular location, and the ability to bind inositol phosphates and other proteins (Harlan et al. 1994; Ingley and Hemmings 1994; Shaw 1996). The presence of the PH domain in MAPK represents a novel adaptation that appears to be specific to oomycetes. The function of the $\mathrm{PH}$ domain in the oomycete MAPK proteins remains unclear. Reminiscent of another report, the pleckstrin domain was also described recently in cellulose synthases of oomycetes, which is apparently also unique (Grenville-Briggs et al. 2008). Notably, oomycetes within this domain have several unique proteins, whereas other organisms do not have the pleckstrin domain integrated. In our phylogenetic analysis, we found that oomycete SAK1s are more closely related to stress-activated MAPK from other species but are grouped together as a distinct clade that is separate from other fungal SAPK.

\section{Functional characterization of PsSAK1.}

The ability of fungi to sense and respond correctly to environmental stress is critical for their survival. One of the most important pathways involved in this response is the SAPK pathway (Gustin et al. 1998; Eaton et al. 2008). Members of this MAPK subfamily are the $S$. cerevisiae Hog1, S. pombe Sty1, Hog1 homologs in other fungi, and mammalian p38s and JNKs. Hog1 is activated by hyperosmotic stress in budding yeast (Brewster et al. 1993). In the fission yeasts and mammals, SAPK respond to multiple stresses such as hyperosmolarity, heat shock, UV light irradiation, and oxidative stress (Waskiewicz and Cooper 1995; Degols et al. 1996; Kato et al. 1996). The function of SAPK in many filamentous fungi is pleiotropic. For example, in rice fungus with OSM1, a stressactivated kinase, the deletion mutants show morphological defects when grown under hyperosmotic conditions; however, glycerol accumulation and turgor generation in appressoria are not altered by the osm1 null mutation (Dixon et al. 1999). In this work, RT-PCR showed that PsSAK1 was differentially expressed during different life stages, and was induced during the early infection process, salt stress, and oxidative treatment, indicating that PsSAK1 is a stress-activated MAPK. After release from sporangia of the PSSAK1-silenced mutants, approximately $20 \%$ of zoospores burst upon release, and this defect could be suppressed with $0.05 \mathrm{M}$ sorbitol solution. Although no differences in the linear growth rate of the PSSAK1-silenced mutants on solid media with different concentration of $\mathrm{NaCl}$ were observed, in the presence of $0.2 \mathrm{M} \mathrm{NaCl}$ the density of mycelium of colony was inhibited. When we introduced hyphal biomass assay to measure the sensitivity to salt stress (Etxebeste et al. 2009), hyphal biomass in the presence of 0.2 $\mathrm{M} \mathrm{NaCl}$ was significantly suppressed in the PsSAK1-silenced mutants. However, in the presence of $0.2 \mathrm{M} \mathrm{NaCl}$, hyphal growth was inhibited. Therefore, the phenotypes are based on a pleiotropic effect of PsSAK1. Because the molecular events that control the osmotic response of Phytophthora spp. are poorly understood, little is known of the molecular mechanism. Examination of which genes can be regulated should to the next goal.

MAPK have been shown to be involved in many essential aspects of fungal pathogenesis and development (Xu 2000; Roman et al. 2007); for example, in Botrytis cinerea, the $B c S A K 1$ deletion mutants are blocked in conidia formation, show increased sclerotial development, and are unable to penetrate unwounded plant tissue (Segmuller et al. 2007) whereas, in Mycosphaerella graminicola, $\mathrm{MgHog} \mathrm{l}$ mutants are unable to switch to filamentous growth on water agar and fail to infect wheat leaves (Mehrabi et al. 2006). Here, we report that, in oomycetes, PsSAK1 is required for zoospore developmental processes such as encystment, germination, and formation of 
appressoria on the host surface, and for pathogenicity. In oomycetes, once released from sporangia, zoospores are motile and chemotactic and may swim for hours under appropriate conditions (Walker and van West 2007). However, silencing of $P S S A K 1$ resulted in rapid encystment of zoospores. This defect should impair chemotaxis because cysts cannot swim. In contrast, PsSAK1 is not associated with mating in $P$. sojae because oospore formation is not disturbed.

As a hemibiotrophic pathogen, $P$. sojae zoospores encyst and germinate on the soybean surface, followed by the growth of infection hyphae or formation of the infection structure, the "appressorium," to penetrate host tissues (Enkerli et al. 1997; Zuo et al. 2005). This silencing of PSSAK1 significantly affects the ability for appressorium formation and osmotic stress response. Compared with the controls, the two PSSAK1-silenced lines were limited in their ability to infect soybean plants in both zoospore and hyphae inoculation assays. The conclusion was made that adaptation of $P$. sojae requires a defense response to be elicited in the host plant during infection. Thus, PSSAK1 plays a crucial role during the infection process, perhaps as part of a signaling network that informs the pathogen of host interactions.

\section{Is PsSAK1 a downstream target of PsGPA1?}

Recently, Hua and associates (2008) found that silencing of the G- $\alpha$-subunit (PsGPA1) in P. sojae resulted in rapid encystment, poor germination, and impaired chemotaxis of zoospores, as well as loss of virulence. Here, we report that the $P S S A K 1$-silenced mutants display a phenotype similar to PsGPA1-silenced mutants, such as rapid encystment, poor germination, and loss of pathogenicity. In fungi, MAPK cascades are often observed to act as downstream targets of heterotrimeric G-proteins (Leberer et al. 1997; Metodiev et al. 2002; Li et al. 2007). The similar expression patterns and mutant phenotypes of PsSAK1 and PsGPAl in P. sojae suggest that PsSAK1 could be one of the downstream targets of PsGPA1. In oomycetes, little is known regarding G-protein signaling pathways. We intend to further explore this hypothesis in our future work.

In conclusion, PSSAK1 functions as a stress-activated MAPK containing a unique structural component ( $\mathrm{PH}$ domain); silencing the PsSAK1 can affect zoospore formation, encystment, germination, and appressorium formation; and more importantly, the PsSAK1-silenced transformants are defective in plant infection, indicating that PsSAK1is involved in the development and pathogenicity of zoospores in $P$. sojae. The signal networks related to development and pathogenicity of Phytophthora pathogens remain unclear. The mutants that we have generated can facilitate exploration of upstream activators and downstream effectors of PsSAK1, and explain how they function in the MAPK signal transduction pathway. The novel MAPK described in this research will also be an ideal target for developing new chemical control molecules and will further help to control this group of notorious plant pathogens.

\section{MATERIALS AND METHODS}

\section{$P$. sojae strains and culture conditions.}

P. sojae strain P6497, provided by B. Tyler (Virginia Bioinformatics Institute, Blacksburg), and all transgenic isolates in this study were routinely grown on $10 \% \mathrm{~V} 8$ media at $25^{\circ} \mathrm{C}$ in the dark as described by Erwin and Ribiero (1996). For growth assays, the recipient strain of P6497 and the silenced lines were subcultured twice, then cultured on $10 \%$ V8 juice agar medium. Radial growth was measured at 4 days. Oospores and zoospores were obtained as described by Erwin and Ribiero
(1996). For the biomass assay, an equal number of hyphal tip plugs from P6497, CK, T31, and T61 were grown in liquid Plich's medium (Lee et al. 1997) for 5, 7, 9, and 11 days. Then, the mycelia were freeze dried and weighed. We collected the asexual samples such as hyphae, sporulating hyphae, zoospores, cysts, and germinated cysts as described by Hua and associates (2008). The samples were immediately frozen in liquid $\mathrm{N}_{2}$ and pulverized for RNA extraction.

Hyphae were cultured in 10\% V8 juice medium for 3 days, washed with sterile distilled water (SDW) three times, and immediately transferred to $50 \mathrm{ml}$ of SDW containing $\mathrm{H}_{2} \mathrm{O}_{2}$ with a concentration of 1 and $4 \mathrm{mM}$ for 10 and $30 \mathrm{~min}$, respectively, or to $50 \mathrm{ml}$ of SDW containing $0.2 \mathrm{M} \mathrm{NaCl}$ for $30 \mathrm{~min}$. Leaf inoculation was performed as described (Chen et al. 2007) at $0,0.5,2,8$, and $12 \mathrm{~h}$. All of the collected samples were immediately frozen in liquid $\mathrm{N}_{2}$ and ground into powder for RNA extraction.

\section{Analysis of zoospore encystment, germination, and appressorium formation.}

To analyze zoospore encystment, equal volumes $(50 \mu \mathrm{l})$ of the zoospore suspension were pipetted onto a glass plate and incubated at $25^{\circ} \mathrm{C}$ with $80 \%$ humidity. The number of encysted zoospores was counted after $0,0.5,2,6$, and $8 \mathrm{~h}$.

To analyze the germination of zoospores, tubes containing $300 \mu \mathrm{l}$ of zoospore suspension were vortexed for $60 \mathrm{~s}$ to induce encystment. Germination was measured by strongly shaking the tubes, then transferring drops of cyst suspension to glass plates and incubating them in 5\% V8 liquid with $80 \%$ humidity at $25^{\circ} \mathrm{C}$ for $0,2,4,6$, and $12 \mathrm{~h}$.

All experiments were repeated independently at least two times.

For appressorium examination, the zoospore suspension was placed on soybean leaves and incubated in a climate room at $25^{\circ} \mathrm{C}$ under $80 \%$ humidity for $4 \mathrm{~h}$. The leaves were destained with alcohol and washed with SDW three or five times. The appressorium formation was examined under the microscope. At least 100 cysts were observed for both P6497 and T31.

\section{Gene identification and sequence analysis.}

$P$. sojae, $P$. ramorum, and $P$. capsici DNA and protein sequence predictions were obtained from the Joint Genome Institute; $P$. infestans DNA and protein sequence was available from the Broad Institute. The $H$. arabidopsis genome sequence was available online. Protein sequences of other SAPK such as Hog1, Sty1, Osm1, and SakA were obtained from the protein databases at the National Center for Biotechnology Information (NCBI). BLAST searches were performed against the above-mentioned genome sequence databases. The PSSAKI sequence was also determined through manual revision on the basis of sequence conservation and dual phosphorylation motif (TxY) (Kultz 1998) and rechecked by submitting to Prosite pattern available on the Internet for conserved function-discriminating residues. The gene PSSAK1, which was highly homologous to Hog1 or other SAPK, was determined.

The orthologs of PsSAK1 in other sequenced Phytophthora genomes, including $P$. ramorum, $P$. infestans, and $P$. capsici, were identified using TBALSTN algorithm. Conserved domain searches were performed using the NCBI CDD search program, and MAPK phosphoylation sites were predicted by CBS NetPhosK 1.0 Server (Blom et al. 2004). Other MAPK sequences used for phylogenetic analysis were obtained from the protein databases at the NCBI. Alignments were made with ClustalX and phylogenetic dendrograms were constructed by MEGA 4.0 (Tamura et al. 2007), with the neighbor-joining algorithms using 1,000 bootstrap replications. 
Plasmid construction and $P$. sojae transformation.

The full-length of PSSAK1 ORF was amplified with PrimeStar polymerase (TaKaRa) from gDNA with oligonucleotides SAK1F1 (5'-atgagctcgcgcggagcttca- $\left.3^{\prime}\right)$ and SAK1R1 (5'-ttat gcgacttcacgtgccg- $\left.3^{\prime}\right)$. This fragment was ligated in antisense orientation into the pHam 34 vector digested by SmaI, and sequenced to confirm the accuracy of the whole ORF. The resulting construct was named pSAK1.

We transformed $P$. sojae by PEG-mediated protoplast transformation (Dou et al. 2008) with a 1:3 combination of the selection plasmids pHSPN and the target plasmids. The vector pHSPN consists of the $h s p 70$ promoter of Bremia lactucea fused to the $n p t I I$ coding sequence and ham 34 terminator of $B$. lactucea (Judelson et al. 1991), and the target plasmids included antisense construct of PSSAK1.

Putative $P$. sojae PSSAKl-silenced transformants were screened as follows. First, genomic PCR screening of all transformant and WT strains was performed with oligonucleotides HMF (5'-ttctccttttcactctcacg-3') and HMR (5'-agacacaaaatctgc aacttc- $\left.3^{\prime}\right)$. Next, RT-PCR was performed on RNA extracted from each line using oligonucleotides SAK1F3 and SAK1R3. Finally, the Southern blot hybridization analysis of transformants and WT was performed.

\section{DNA and RNA manipulation of $P$. sojae.}

gDNA of $P$. sojae was isolated from hyphae grown in $10 \%$ V8 liquid medium following the protocol described by Tyler and associates (1995). DNA blots were performed using digoxigenin (DIG) kit I (Roche Applied Science 11745832 910). The probe of $P S S A K 1$ was amplified from pSAK1 with the primers SAK1F2 (5'-agctcaagatctgcgactttg- $\left.3^{\prime}\right)$ and SAK1R2 (5'-gtctgAgcgtctgtcgggttg-3') and confirmed by sequencing, then labeled by DIG-dUTP alkali-labile. Samples of $5 \mu \mathrm{g}$ of gDNA of P6497 or PsSAK1-silenced transformants were digested with selected restriction enzymes prior to DNA blot analysis.

Total RNA was isolated using the NucleoSpin RNA II RNA extraction kit (Macherey-Nagel) following the procedures described by the manufacturer. The integrity of total RNA was confirmed using agarose gel electrophoresis.

To investigate the efficiency of $P S S A K l$ gene silencing in the putative transformants, semiquantitative RT-PCR was performed according to the following steps. First-strand cDNA was synthesized using M-MLV reverse transcriptase (RNasefree) and oligo(dT) 18 primer (Invitrogen); PCR for PSSAK1 was amplified with the primers of SAK1F3 $\left(5^{\prime}\right.$-actacacaggca tccaacacc-3') and SAK1R3 (5'-ttactgccgaccttccacgctgcccg-3') under the following conditions: $94^{\circ} \mathrm{C}$ for $1 \mathrm{~min}$; followed by 30 cycles of $94^{\circ} \mathrm{C}$ for $30 \mathrm{~s}, 52^{\circ} \mathrm{C}$ for $30 \mathrm{~s}$, and $72^{\circ} \mathrm{C}$ for $30 \mathrm{~s}$; and a final extension of $72^{\circ} \mathrm{C}$ for $10 \mathrm{~min}$. The $P$. sojae actin A (ActA) gene was used as reference with PCR conditions of $94^{\circ} \mathrm{C}$ for $1 \mathrm{~min}$; followed by 24 cycles of $94^{\circ} \mathrm{C}$ for $30 \mathrm{~s}, 59^{\circ} \mathrm{C}$ for $30 \mathrm{~s}$, and $72^{\circ} \mathrm{C}$ for $30 \mathrm{~s}$; and a final extension of $72^{\circ} \mathrm{C}$ for $10 \mathrm{~min}$ with the primers of actAF $\left(5^{\prime}\right.$-gtactgcaacatcgtgctgtcg$\left.3^{\prime}\right)$ and actAR (5'-ttagaagcacttgcggtgcacg- $\left.3^{\prime}\right)$. The absence of contaminating gDNA was validated by PCR, using the same conditions as those used for the RT-PCR, except that the cDNA synthesis step at $37^{\circ} \mathrm{C}$ was omitted.

\section{Virulence test and microscopic observation.}

For infection assays, detached soybean leaves of Williams, a cultivar susceptible to most $P$. sojae strains, including P6497, were placed in petri dishes. Each leaflet was inoculated on the abaxial side with a water droplet containing a suspension of $10^{3}$ effective zoospores preserving germination ability according to the cyst germination ratio at $4 \mathrm{~h}$. The leaves were incubated in a climate room at $25^{\circ} \mathrm{C}$ under $80 \%$ humidity for
5 days. In wounded assays, leaves were abraded by scratching with a pipette tip before inoculation. Pictures were taken 5 dpi.

Inoculation of etiolate soybean hypocotyls with $P$. sojae hyphal tip plugs or zoospore suspensions was carried out as described by Qutob and associates (2002) with a little modification (Moy et al. 2004). The hyphal tip plugs or zoospore suspensions of P6497, CK, T31, and T61 were placed on hypocotyls of etiolate soybean cv. Williams. Each treatment contained at least 10 hypocotyls. All treated seedlings were incubated in the dark at $25^{\circ} \mathrm{C}$ under $80 \%$ humidity for at least $36 \mathrm{~h}$. The virulence tests were repeated at least four times, independently.

Hypocotyls of 4-day-old soybean cv. Williams, growing without light, were spot incubated with equal numbers of zoospores of P6497, CK, T31, and T61 for $6 \mathrm{~h}$. Then, the epidermis tissue around the inoculation site was peeled off and used for microscopic observation.

Excised leaflets inoculated with zoospores for $24 \mathrm{~h}$ were put into alcohol to destain, then soaked in $0.5 \%$ Coomassie brilliant blue for $2 \mathrm{~min}$. After the leaves were washed in SDW for $10 \mathrm{~min}$, the microscopic pictures were taken.

\section{ACKNOWLEDGMENTS}

We thank M. Gijzen (Agriculture and Agri-Food Canada, Ontario) for critically reading this manuscript. This work was supported by the National "973" project (2009CB119202), the NSFC project (30671345), and Commonweal Specialized Research Fund of China Agriculture (3-20).

\section{LITERATURE CITED}

Banuett, F. 1998. Signalling in the yeasts: an informational cascade with links to the filamentous fungi. Microbiol. Mol. Biol. Rev. 62:249274.

Blanco, F. A., and Judelson, H. S. 2005. A bZIP transcription factor from Phytophthora interacts with a protein kinase and is required for zoospore motility and plant infection. Mol. Microbiol. 56:638-648.

Blom, N., Sicheritz-Ponten, T., Gupta, R., Gammeltoft, S., and Brunak, S. 2004. Prediction of post-translational glycosylation and phosphorylation of proteins from the amino acid sequence. Proteomics 4:16331649.

Brewster, J. L., de Valoir, T., Dwyer, N. D., Winter, E., and Gustin, M. C. 1993. An osmosensing signal transduction pathway in yeast. Science 259:1760-1763.

Chen, X., Shen, G., Wang, Y., Zheng, X., and Wang, Y. 2007. Identification of Phytophthora sojae genes upregulated during the early stage of soybean infection. FEMS (Fed. Eur. Microbiol. Soc.) Microbiol. Lett. 269:280-288.

Chen, X., Wang, X., Zhang, Z., Wang, Y., and Zheng, X. 2008. Differences in the induction of the oxidative burst in compatible and incompatible interactions of soybean and Phytophthora sojae. Physiol. Mol. Plant Pathol. 73:16-24

Degols, G., Shiozaki, K., and Russell, P. 1996. Activation and regulation of the Spc1 stress-activated protein kinase in Schizosaccharomyces pombe. Mol. Cell Biol. 16:2870-2877.

Dixon, K. P., Xu, J. R., Smirnoff, N., and Talbot, N. J. 1999. Independent signaling pathways regulate cellular turgor during hyperosmotic stress and appressorium-mediated plant infection by Magnaporthe grisea. Plant Cell 11:2045-2058.

Dou, D., Kale, S. D., Wang, X., Chen, Y., Wang, Q., Jiang, R. H., Arredondo, F. D., Anderson, R. G., Thakur, P. B., McDowell, J. M., Wang, Y., and Tyler, B. M. 2008. Conserved C-terminal motifs required for avirulence and suppression of cell death by Phytophthora sojae effector Avr1b. Plant Cell 20:1118-1133.

Eaton, C. J., Jourdain, I., Foster, S. J., Hyams, J. S., and Scott, B. 2008. Functional analysis of a fungal endophyte stress-activated MAP kinase. Curr. Genet. 53:163-174.

Enkerli, K., Hahn, M., and Mims, C. 1997. Ultrastructure of compatible and incompatible interactions of soybean roots infected with the plant pathogenic oomycete Phytophthora sojae. Can. J. Bot. 75:1493-1508.

Erwin, D. C., and Ribiero, O. K. 1996. Phytophthora Diseases Worldwide. American Phytopathological Society Press, St. Paul, MN, U.S.A.

Etxebeste, O., Herrero-Garcia, E., Araujo-Bazan, L., Rodríguez-Urra, A. 
B., Garzia, A., Ugalde, U., and Espeso, E. A. 2009. The bZIP-type transcription factor FlbB regulates distinct morphogenetic stages of colony formation in Aspergillus nidulans. Mol Microbiol 73:775-789.

Förster, H., Coffey, M. D., Elwood, H., and Sogin, M. L. 1990. Sequence analysis of the small subunit ribosomal RNAs of three zoosporic fungi and implications for fungal evolution. Mycologia 82:306312

Grenville-Briggs, L. J., Anderson, V. L., Fugelstad, J., Avrova, A. O., Bouzenzana, J., Williams, A., Wawra, S., Whisson, S. C., Birch, P. R., Bulone, V., and van West, P. 2008. Cellulose synthesis in Phytophthora infestans is required for normal appressorium formation and successful infection of potato. Plant Cell 20:720-738.

Gustin, M. C., Albertyn, J., Alexander, M., and Davenport, K. 1998. MAP kinase pathways in the yeast Saccharomyces cerevisiae. Microbiol. Mol. Biol. Rev. 62:1264-1300.

Harlan, J. E., Hajduk, P. J., Yoon, H. S., and Fesik, S. W. 1994. Pleckstrin homology domains bind to phosphatidylinositol-4,5-bisphosphate. Nature 371:168-170.

Harper, J. T., Waanders, E., and Keeling, P. J. 2005. On the monophyly of chromalveolates using a six-protein phylogeny of eukaryotes. Int. J. Syst. Evol. Microbiol. 55:487-496.

Herskowitz, I. 1995. MAP kinase pathways in yeast: for mating and more Cell 80:187-197.

Hohmann, S. 2002. Osmotic stress signaling and osmoadaptation in yeasts. Microbiol. Mol. Biol. Rev. 66:300-372.

Hua, C., Wang, Y., Zheng, X., Dou, D., Zhang, Z., Govers, F., and Wang, Y. 2008. A Phytophthora sojae G-protein $\alpha$ subunit is involved in chemotaxis to soybean isoflavones. Eukaryot. Cell 7:2133-2140.

Ingley, E., and Hemmings, B. A. 1994. Pleckstrin homology (PH) domains in signal transduction. J. Cell. Biochem. 56:436-443.

Judelson, H. S., and Roberts, S. 2002. Novel protein kinase induced during sporangial cleavage in the oomycete Phytophthora infestans. Eukaryot. Cell 1:687-695.

Judelson, H. S., Tyler, B. M., and Michelmore, R. W. 1991. Transformation of the oomycete pathogen, Phytophthora infestans. Mol. Plant-Microbe Interact. 4:602-607.

Kato, T., Jr., Okazaki, K., Murakami, H., Stettler, S., Fantes, P. A., and Okayama, H. 1996. Stress signal, mediated by a Hog1-like MAP kinase, controls sexual development in fission yeast. FEBS (Fed. Eur. Biochem. Soc.) Lett. 378:207-212.

Kultz, D. 1998. Phylogenetic and functional classification of mitogen- and stress-activated protein kinases. J. Mol. Evol. 46:571-588.

Kyriakis, J. M., and Avruch, J. 1996. Sounding the alarm: protein kinase cascades activated by stress and inflammation. J. Biol. Chem. 271:24313-24316.

Kyriakis, J. M., and Avruch, J. 2001. Mammalian mitogen-activated protein kinase signal transduction pathways activated by stress and inflammation. Physiol. Rev. 81:807-869.

Latijnhouwers, M., Munnik, T., and Govers, F. 2002. Phospholipase D in Phytophthora infestans and its role in zoospore encystment. Mol. PlantMicrobe Interact. 15:939-946.

Latijnhouwers, M., Ligterink, W., Vleeshouwers, V. G., van West, P., and Govers, F. 2004. A Galpha subunit controls zoospore motility and virulence in the potato late blight pathogen Phytophthora infestans. Mol. Microbiol. 51:925-936.

Leberer, E., Thomas, D. Y., and Whiteway, M. 1997. Pheromone signalling and polarized morphogenesis in yeast. Curr. Opin. Genet. Dev. 7:59-66.

Lee, V. D., Finstad, S. L., and Huang, B. 1997. Cloning and characterization of a gene encoding an actin-related protein in Chlamydomonas. Gene 197:153-159.

Li, L., Wright, S. J., Krystofova, S., Park, G., and Borkovich, K. A. 2007. Heterotrimeric $G$ protein signaling in filamentous fungi. Annu. Rev. Microbiol. 61:423-452.

Mehrabi, R., Zwiers, L. H., de Waard, M. A., and Kema, G. H. 2006 $\mathrm{MgHog} 1$ regulates dimorphism and pathogenicity in the fungal wheat pathogen Mycosphaerella graminicola. Mol. Plant-Microbe Interact. 19:1262-1269.

Metodiev, M. V., Matheos, D., Rose, M. D., and Stone, D. E. 2002. Regulation of MAPK function by direct interaction with the mating-specific Galpha in yeast. Science 296:1483-1486.

Millar, J. B., Buck, V., and Wilkinson, M. G. 1995. Pyp1 and Pyp2 PTPases dephosphorylate an osmosensing MAP kinase controlling cell size at division in fission yeast. Genes Dev. 9:2117-2130.

Moriwaki, A., Kubo, E., Arase, S., and Kihara, J. 2006. Disruption of SRM1, a mitogen-activated protein kinase gene, affects sensitivity to osmotic and ultraviolet stressors in the phytopathogenic fungus Bipolaris oryzae. FEMS (Fed. Eur. Microbiol. Soc.) Microbiol. Lett. 257:253-261.

Morris, P. F., Bone, E., and Tyler, B. M. 1998. Chemotropic and contact responses of Phytophthora sojae hyphae to soybean isoflavonoids and artificial substrates. Plant Physiol. 117:1171-1178.
Moy, P., Qutob, D., Chapman, B. P., Atkinson, I., and Gijzen, M. 2004. Patterns of gene expression upon infection of soybean plants by Phytophthora sojae. Mol. Plant-Microbe Interact. 17:1051-1062.

Park, S. M., Choi, E. S., Kim, M. J., Cha, B. J., Yang, M. S., and Kim, D. H. 2004. Characterization of HOG1 homologue, CpMK1, from Cryphonectria parasitica and evidence for hypovirus-mediated perturbation of its phosphorylation in response to hypertonic stress. Mol. Microbiol. 51:1267-1277.

Rebecchi, M. J., and Scarlata, S. 1998. Pleckstrin homology domains: a common fold with diverse functions. Annu. Rev. Biophys. Biomol. Struct. 27:503-528.

Roman, E., Arana, D. M., Nombela, C., Alonso-Monge, R., and Pla, J. 2007. MAP kinase pathways as regulators of fungal virulence. Trends Microbiol. 15:181-190.

Qutob, D., Kamoun, S., and Gijzen, M. 2002. Expression of a Phytophthora sojae necrosis-inducing protein occurs during transition from biotrophy to necrotrophy. Plant J. 32:361-373.

Schuller, C., Brewster, J. L., Alexander, M. R., Gustin, M. C., and Ruis, H. 1994. The HOG pathway controls osmotic regulation of transcription via the stress response element (STRE) of the Saccharomyces cerevisiae CTT1 gene. EMBO (Eur. Mol. Biol. Organ.) J. 13:43824389 .

Segmuller, N., Ellendorf, U., Tudzynski, B., and Tudzynski, P. 2007. BcSAK1, a stress-activated mitogen-activated protein kinase, is involved in vegetative differentiation and pathogenicity in Botrytis cinerea. Eukaryot. Cell 6:211-221.

Shaw, G. 1996. The pleckstrin homology domain: an intriguing multifunctional protein module. Bioessays 18:35-46.

Shen, W., Gomez-Cadenas, A., Routly, E. L., Ho, T. H., Simmonds, J. A., and Gulick, P. J. 2001. The salt stress-inducible protein kinase gene, Esi47, from the salt-tolerant wheatgrass Lophopyrum elongatum is involved in plant hormone signaling. Plant Physiol. 125:14291441.

Shiozaki, K., and Russell, P. 1995. Cell-cycle control linked to extracellular environment by MAP kinase pathway in fission yeast. Nature 378:739-743.

Sogin, M. L., and Silberman, J. D. 1998. Evolution of the protists and protistan parasites from the perspective of molecular systematics. Int. J. Parasitol. 28:11-20.

Tamura, K., Dudley, J., Nei, M., and Kumar, S. 2007. MEGA4: Molecular Evolutionary Genetics Analysis (MEGA) software version 4.0. Mol. Biol. Evol. 24:1596-1599.

Treisman, R. 1996. Regulation of transcription by MAP kinase cascades. Curr. Opin. Cell Biol. 8:205-215.

Tyler, B. M. 2007. Phytophthora sojae: root rot pathogen of soybean and model oomycete. Mol. Plant Pathol. 8:1-8.

Tyler, B. M., Forster, H., and Coffey, M. D. 1995. Inheritance of avirulence factors and restriction fragment length polymorphism Markersin outcrosses of the oomycete Phytophthora sojae. Mol. Plant-Microbe Interact. 8:515-523.

Tyler, B. M., Wu, M., Wang, J., Cheung, W., and Morris, P. F. 1996. Chemotactic preferences and strain variation in the response of Phytophthora sojae zoospores to host isoflavones. Appl. Environ. Microbiol. 62:2811-2817.

Tyler, B. M., Tripathy, S., Zhang, X., Dehal, P., Jiang, R. H., Aerts, A., Arredondo, F. D., Baxter, L., Bensasson, D., Beynon, J. L., Chapman, J., Damasceno, C. M., Dorrance, A. E., Dou, D., Dickerman, A. W., Dubchak, I. L., Garbelotto, M., Gijzen, M., Gordon, S. G., Govers, F., Grunwald, N. J., Huang, W., Ivors, K. L., Jones, R. W., Kamoun, S., Krampis, K., Lamour, K. H., Lee, M. K., McDonald, W. H., Medina, M., Meijer, H. J., Nordberg, E. K., Maclean, D. J., Ospina-Giraldo, M. D., Morris, P. F., Phuntumart, V., Putnam, N. H., Rash, S., Rose, J. K., Sakihama, Y., Salamov, A. A., Savidor, A., Scheuring, C. F., Smith, B. M., Sobral, B. W., Terry, A., Torto-Alalibo, T. A., Win, J., Xu, Z., Zhang, H., Grigoriev, I. V., Rokhsar, D. S., and Boore, J. L. 2006. Phytophthora genome sequences uncover evolutionary origins and mechanisms of pathogenesis. Science 313:1261-1266.

Walker, C. A., and van West, P. 2007. Zoospore development in the oomycetes. Fungal Biol. Rev. 21:10-18.

Walker, C. A., Koppe, M., Grenville-Briggs, L. J., Avrova, A. O., Horner, N. R., McKinnon, A. D., Whisson, S. C., Birch, P. R., and van West, P. 2008. A putative DEAD-box RNA-helicase is required for normal zoospore development in the late blight pathogen Phytophthora infestans. Fungal Genet. Biol. 45:954-962.

Waskiewicz, A. J., and Cooper, J. A. 1995. Mitogen and stress response pathways: MAP kinase cascades and phosphatase regulation in mammals and yeast. Curr. Opin. Cell. Biol. 7:798-805.

Winkler, A., Arkind, C., Mattison, C. P., Burkholder, A., Knoche, K., and Ota, I. 2002. Heat stress activates the yeast high-osmolarity glycerol mitogen-activated protein kinase pathway, and protein tyrosine phos- 
phatases are essential under heat stress. Eukaryot. Cell 1:163-173.

Wrather, J. A., and Koenning, S. R. 2006. Estimates of disease effects on soybean yields in the United States 2003 to 2005. J. Nematol. 38:173180

Xu, J. R. 2000. Map kinases in fungal pathogens. Fungal Genet. Biol 31:137-152.

Yoshioka, H., Numata, N., Nakajima, K., Katou, S., Kawakita, K., Rowland, O., Jones, J. D., and Doke, N. 2003. Nicotiana benthamiana gp91 ${ }^{\text {phox }}$ homologs $N b r b o h A$ and $N b r b o h B$ participate in $\mathrm{H}_{2} \mathrm{O}_{2}$ accumulation and resistance to Phytophthora infestans. Plant Cell 15:706-718.

Zuo, Y., Kang, Z., Huang, L., and Han, Q. 2005. Cytology on infection process of soybean hypocotyls by Phytophthora sojae. Acta Phytopathol. Sin. 35:235-241.
AUTHOR-RECOMMENDED INTERNET RESOURCES

Broad Institute website: www.broad.mit.eduJoint Genome Institute website: www.jgi.doe.gov

National Center for Biotechnology Information (NCBI) website: www.ncbi.nlm.nih.gov

NCBI conserved domains database: www.ncbi.nlm.nih.gov/Structure/cdd/wrpsb.cgi

Center for Biological Sequence Analysis NetPhosK 1.0 Server: www.cbs.dtu.dk/services/NetPhosK

ExPasy Proteomics Server Prosite database: www.expasy.org/prosite

Virginia Bioinformatics Institute Phytophthora EST database: vmd.vbi.vt.edu 